\title{
Strain rate dependency of laser sintered polyamide 12
}

\author{
J.E.T. Cook ${ }^{1, \text { a }}$, R.D. Goodridge ${ }^{2}$, and C.R. Siviour ${ }^{1}$ \\ ${ }^{1}$ Department of Engineering Science, University of Oxford, Parks Road, Oxford, UK \\ ${ }^{2}$ Additive Manufacturing \& 3D-Printing Research Group, School of Engineering, University of Nottingham, Nottingham, \\ UK
}

\begin{abstract}
Parts processed by Additive Manufacturing can now be found across a wide range of applications, such as those in the aerospace and automotive industry in which the mechanical response must be optimised. Many of these applications are subjected to high rate or impact loading, yet it is believed that there is no prior research on the strain rate dependence in these materials. This research investigates the effect of strain rate and laser energy density on laser sintered polyamide 12. In the study presented here, parts produced using four different laser sintered energy densities were exposed to uniaxial compression tests at strain rates ranging from $10^{-3}$ to $10^{+3} \mathrm{~s}^{-1}$ at room temperature, and the dependence on these parameters is presented.
\end{abstract}

\section{Introduction}

The principle advantage of Additive Manufacturing $(\mathrm{AM})$ is the ability to form complex geometries that would be impossible or highly expensive compared to conventional subtractive or formative methods. In Additive Manufacturing objects are directly built from CAD models by depositing material in a layer-by-layer process. Laser sintering (LS) is a form of AM whereby a thin layer of powder material is deposited onto a pre-heated build area (normally pre-heated), a CO2 laser is then used to selectively consolidate the powder. The consolidated regions form the final product, whilst the remaining powder is removed.

LS is increasingly being used in demanding applications, with the aviation and F1 industry adopting LS for processing parts [1]. Detailed analysis of the stiffness, strength and surface finish must be further understood for LS parts to meet future in-service loading and operational requirements. The performance of components under high rate and impact loading is a particularly important design criterion for crash worthiness.

The mechanical properties of LS can in part be attributed to the degree of particle melt (DPM) [2], which defines the quantitative variations in the consolidation of sintering. Powder properties (e.g. morphology) and build parameters (e.g. laser energy, density, temperature) can affect the DPM of a given material in laser sintering [3].

Differential scanning calorimetry (DSC) is frequently used to predict the DPM of a material [3]. However, it is believed that there is no prior research on the dependency of strain rate on a part's mechanical properties. By understanding the effect of a wide range of strain rates it is hoped that a more versatile constitutive model for LS components can be developed. A range of energy densities are investigated to help determine the particular effect of

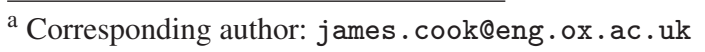

strain rate, where it is hoped that this will provide further information on the role of the microstructure.

\subsection{Calculating the energy density}

Also known as the Andrew number, it has been shown that the energy per unit area supplied by the laser to the powder bed is dependent on the laser power (W), scan speed $(\mathrm{mm} / \mathrm{s})$ and scan spacing $(\mathrm{mm})$ [4].

$$
\begin{aligned}
& E D\left(\mathrm{~J} / \mathrm{mm}^{2}\right)= \\
& \quad \times \text { Laser power } /\left(\text { scan speed }{ }^{*} \text { scan spacing }\right) .
\end{aligned}
$$

\subsection{Effect of the energy density}

The energy applied to the powder causes coalescence by tipping the material into its molten state. The typical $\mathrm{T}_{\mathrm{M}}$ for polyamide 12 is $175^{\circ} \mathrm{C}$. The powder bed is heated to approximately $152^{\circ} \mathrm{C}$ to prevent rapid cooling of the part. As polyamide 12 is a semi-crystalline polymer, rapidly cooling through its glass transition will cause significant shrinkage and inherent thermal stress concentrations.

In reality, powder cannot be uniformly consolidated due to limitations of the scan spacing, precision of levelled powder and variation in powder morphology. In partial melting only the shell of the powder melts allowing the molten polymer to form necks between adjacent powders. However, the core can remain solid which is characterised by an un-molten core surrounded by spherulites.

By imparting a different energy density one can alter the inter-crystal microstructure through (a) powder coalescence (necking of particles) (b) and crystallised particles (spherulites) [2]. By investigated various energy densities, it is possible to better understand the role of a) and b) on mechanical properties.

\section{Experimental approach}

Four different energy densities were investigated in this study, listed in Table 1. Only the laser power and scan 
Table 1. Energy densities investigated.

\begin{tabular}{|l|l|l|l|l|}
\hline & ED1 & ED2 & ED3 & ED4 \\
\hline Laser Power $(\mathrm{W})$ & 19 & 21 & 23 & 21 \\
\hline Scan spacing $(\mathrm{mm})$ & 0.25 & 0.25 & 0.25 & 0.25 \\
\hline Scan speed $(\mathrm{mm} / \mathrm{s})$ & 2500 & 2500 & 2500 & 1500 \\
\hline Energy Density $\left(\mathbf{J} / \mathbf{m m}^{2}\right)$ & $\mathbf{0 . 0 3}$ & $\mathbf{0 . 0 3 4}$ & $\mathbf{0 . 0 3 7}$ & $\mathbf{0 . 0 5 6}$ \\
\hline Physical density $\left(\mathbf{k g} / \mathbf{m}^{\mathbf{3}}\right)$ & $\mathbf{8 8 9}$ & $\mathbf{8 7 6}$ & $\mathbf{8 7 3}$ & $\mathbf{8 8 4}$ \\
\hline
\end{tabular}

speed was varied. Each energy density was used to form a $6 \times 6 \mathrm{~mm}$ right cylinder, where a range of strain rates were applied to investigate the relationship between strain rate and relative density in compression. All samples were processed on an EOS P100 machine. Each sample was orientated in the $\mathrm{z}$-axis, which will be the subsequent loading axis, during part build.

The mass and volume of each energy density was measured five times to calculate the respective mean physical density, also listed in Table 1. The mass was found using a XS105 dualrange analytical balance with a readability of $0.01 \mathrm{mg}$. A micrometer of readability $0.001 \mathrm{~mm}$ was used to gauge the length, height and width of each cube specimen.

The quasi-static compression testing was undertaken using an Instron testing machine. For these low strength materials machine compliance is not an issue, and true strain control from the cross head was used however, an extensometer was also attached to the loading anvils close to the specimen to verify the specimen extension. The total resisting force on the specimen as a function of time was obtained from a $100 \mathrm{kN}$ load cell with a stated precision of $\pm 0.5 \mathrm{~N}$.

Medium strain rate was obtained through a custom built hydraulic load frame that is used to access strain rates between 1 and $50 \mathrm{~s}^{-1}$. A linear Variable Differential Transformer (LVDT) measured the displacement of the sample; the signal suffered no significant distortion from load cell ringing and other machine noise.

High strain rate compression experiments were performed using a Split Hopkinson Pressure Bar (SHPB). For the SHPB system, the input and output bars were made of silver steel. The input bar is $1 \mathrm{~m}$ long, and gauged halfway along its length; the output bar is $500 \mathrm{~mm}$ long and gauged $50 \mathrm{~mm}$ from the bar-specimen interface. The reflected and transmitted gauge signals are used to derive the stress strain relationship using the standard analysis [6].

To obtain reliable data, good lubrication of the anvils is essential. A control test of lubricants for the semicrystalline polymer - Polypropylene was investigated by Okereke [7]. In the post-yield region, petroleum jelly gave the lower yield/flow stress indicating that least friction was generated and so this lubricant was applied for all testing in the current study.

Barrelling of the sample was not apparent post compression. However, occasionally slanting was seen, in which one end of the recovered specimen was observed to have a larger diameter than the other. This was connected to defects of the cell wall during the LS process.

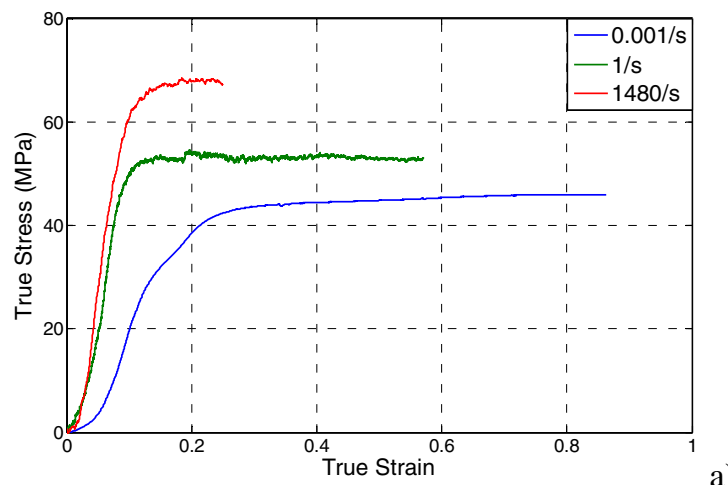

a)

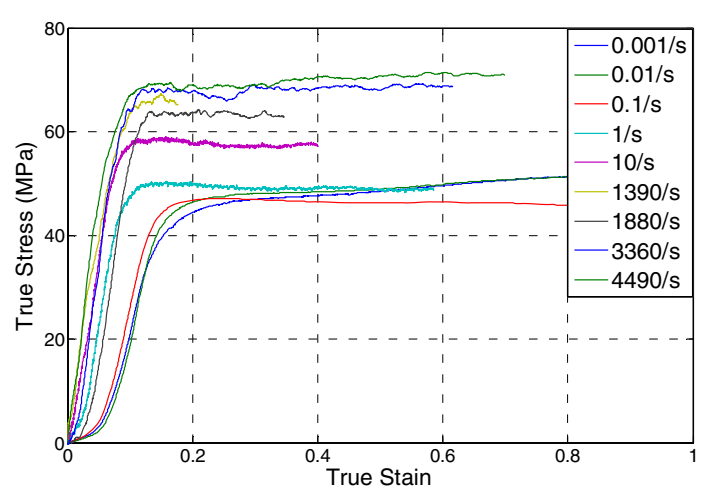

b)

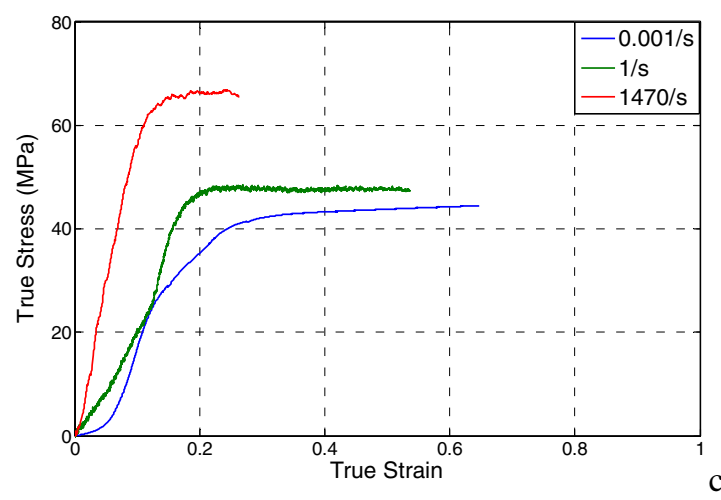

c)

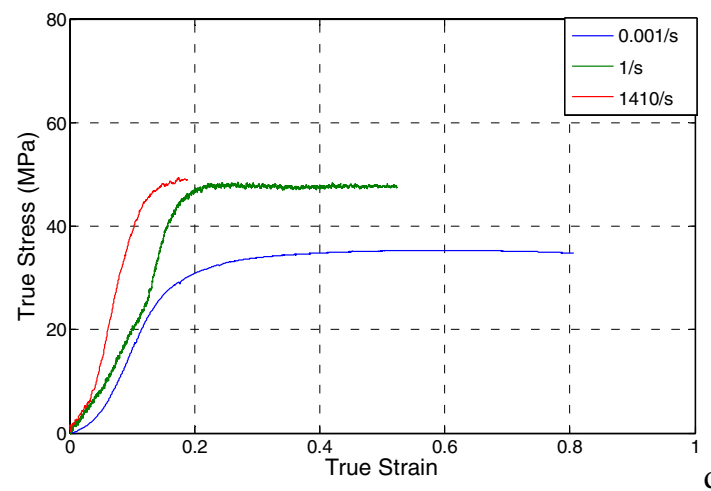

Figure 1. Compressive stress-strain response of ED, from top: a) $0.030 \mathrm{~J} / \mathrm{mm}^{2}$ b) $0.034 \mathrm{~J} / \mathrm{mm}^{2}$ c) $0.037 \mathrm{~J} / \mathrm{mm}^{2}$ d) $0.056 \mathrm{~J} / \mathrm{mm}^{2}$.

\section{Results and discussion}

The stress-strain response of four different energy densities can be seen in Fig. 1, where a wide range of strain rates are applied in uniaxial compression. Results are plotted astrue 


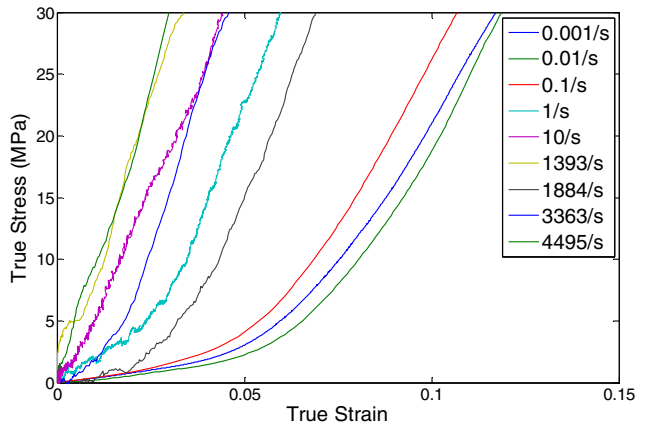

Figure 2. Effect of surface roughness for $0.034 \mathrm{~J} / \mathrm{mm}^{2}$ over a range of strain rate.

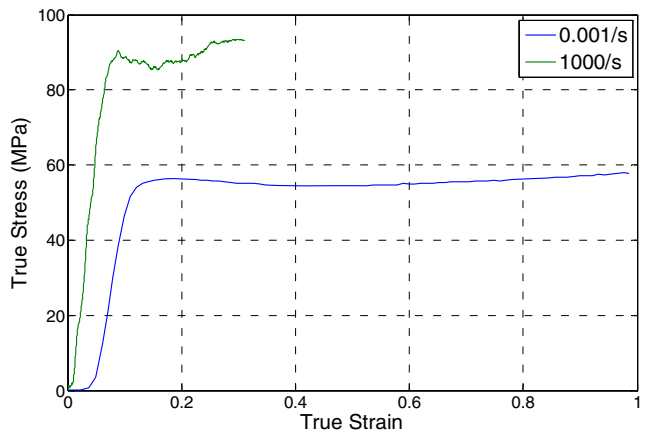

Figure 3. Extruded polyamide 12 at low and high strain rates.

strain against true stress. The true strain is determined by assuming constant volume. Whilst three of the energy densities were tested at rates c.a. $0.001,1$ and $1500 \mathrm{~s}^{-1}$, one energy density, $0.0034 \mathrm{~J} / \mathrm{mm}^{2}$, which is currently the most commonly used, was tested over a wide range of rates, Fig. 1(b).

The point at which yield stress is initiated is seen to shift to a lower true strain as the strain rate increases for ED1, 2 and 3, particularly between low and medium strain rates. The shift is unusual compared with other semicrystalline polymers such as polypropylene, where yield stress is observed to start at $10 \%$ true strain across all strain rates (see e.g. [7]). Looking at Fig. 2, it was observed that this is due to a low strength 'bedding in' before the main deformation. This is attributed to surface roughess in the specimen, which is either more easily observed, or has a greater effect, in the quasi-static experiments.

The LS compression tests can be compared to the mechanical response of extruded polyamide 12 at low and high rate, which is shown in Fig. 3.

Compared to the data from the SL material, the extruded polyamide shows a small amount of strain softening after yield. This is common for glassy polymers. A mechanism developed by Boyce and Haward showed that plastic strain-induced structural evolution of the glass can create a less compact but higher energy structure that produces lower flow stresses. [8].

This phenomenon is characteristic of a glassy polymer deformed at a temperature below its glass transition, and causes post yield softening in the material.

In Fig. 3, only slight softening can be seen. However, the degree of strain softening appears to increase from strain rate $10^{-3}$ to $10^{+3} \mathrm{~s}^{-1}$. According to Wang and

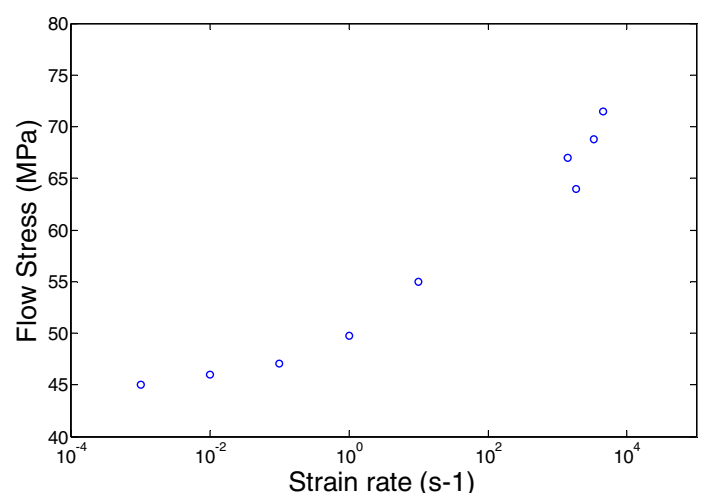

Figure 4. Eyring plot showing stress at $25 \%$ strain vs $\log \dot{\varepsilon}$ for $0.034 \mathrm{~J} / \mathrm{mm}^{2}$.

Arrruda [9], high strain rate deformation can cause strain softening due to adiabatic heating within the sample.

Interestingly, no strain softening or hardening was seen in the LS polymer. The consistent flow stress could be due to the larger degree of porosity in the specimen, making it more akin to a foam, which will require further investigation. LS is known to produce parts that have significant porosity due to partial coalescence of powder, also known as necking. This will be discussed further below.

With a wide range of strain rate testing on the LS sample, it is possible to conduct an Eyring plot, showing the yield stress versus the logarithm of strain rate, as seen in Fig. 4.

Here the flow stress was chosen to be the stress at $25 \%$ strain. The Eyring plot is usually linear for most polymers at room temperature when tested over a modest range of strain rate. However, when constructed over a wide range, many polymers show a significant increase in strain-rate dependence at higher rates and a similar effect is seen here.

This response, often described as bi-linear, has been shown in other polymers due to the transition between two different relaxation times governing the response. These can be assumed to be the $\alpha$-(glass) and $\beta$-processes with $\tau_{\alpha}>\tau_{\beta}$, since the glass temperature is measured in the literature to be $155\left[{ }^{\circ} \mathrm{C}\right]$. The increased strain rate dependence of the yield strength of LS polyamide 12 at high strain rates is therefore expected to be a material property and is due to the movement of the $\beta$ transition to room temperature at these rates. An understanding of this transition is crucial to predicting the material's response to high strain rate deformation [10].

It has been shown that the degree of a) crystallisation and b) coalescence of powder are related to the rate of cooling. A change of energy density will vary the thermal gradient between powder temperature and the temperature of the molten region, the steeper this gradient, the more likely it is that higher rates of cooling will occur [3]. The high rate of cooling will inhibit coalescence of powder causing high porosity in the part. It would be expected that ED4 $=0.056 \mathrm{~J} / \mathrm{mm} 2$ would have the highest thermal gradient and thus the highest level of porosity. However, on measuring the physical densities there was no trend between the physical density and energy density, as shown in Fig. 5 suggesting that the energy density between 0.03 and $0.56 \mathrm{~J} / \mathrm{mm}^{2}$ has little effect on the degree of porosity. 


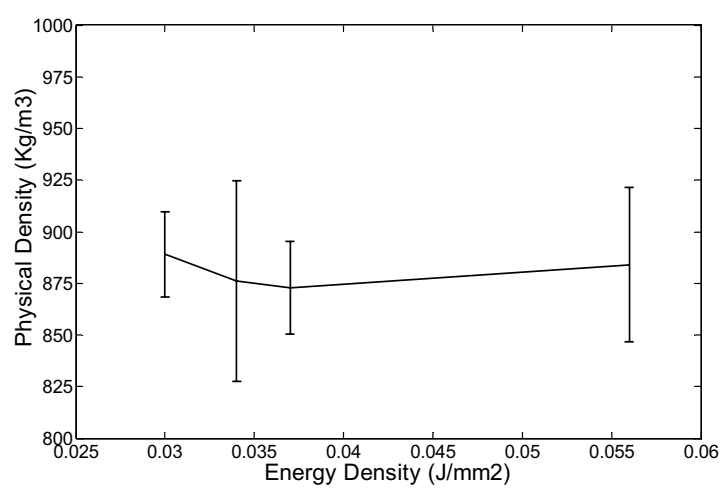

Figure 5. The physical density for four different energy densities. Error bars represent standard deviation from 5 measurements.

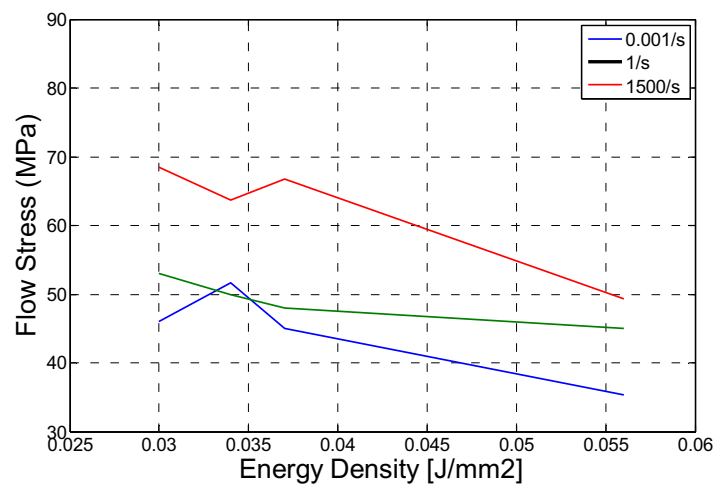

Figure 6. Relationship between energy density and flow stress. Flow stress is obtained at the initial point of plateau.

According to Ashby and Gibson [11] the yield strength for open macro cellular foams can be predicted from the degree of porosity as shown in equation 2 .

$$
\sigma^{*}=C_{1} \sigma_{s}\left(p^{*} / p_{s}\right)
$$

$\mathrm{C}_{1}$ is dependent on the material, $\sigma_{s}$ is the yield strength for fully dense material and $\left(\mathrm{p}^{*} / \mathrm{p}_{s}\right)$ is the relative density of the foam. However, in this case, there was no significant change in physical density between the different specimens, and it is therefore likely that changes in properties between the energy densities are a result of smaller scale microstructural changes in the polymer.

Figure 6 shows the dependence of flow stress on energy density at three strain rates. Because some of the samples were not tested to $25 \%$ strain, the flow stress was obtained at the initial point of plateau. There appears to be a general, but weak, trend of decreasing strength with increasing energy density. However, there is no observed correlation between ED1, ED2, ED3 and their respective flow stresses. One possible cause of energy density dependence could be the change in activation volume at the molecular level due to the degree of crystallinity in the microstructure, which has been shown to vary according to the energy density [2], and may affect the activation volume and hence the rate dependence.

\section{Conclusions}

AM allows the construction of more complex geometries than traditional manufacturing techniques, giving designers and engineers the freedom to further optimise parts. AM lattices are used in the automotive industry as they provide an enhanced mechanical response in impact absorption. This paper has two objectives; firstly, to investigate the strain rate dependency of polyamide 12 , and secondly to further understand the relationship between energy density and strain rate.

The flow stress was found to be consistent after yield, showing no strain softening or strain hardening. The plateau of flow stress can be related to the porosity as observed in foams and under SEM of polyamide 12 [12]. The apparent true strain at which yield stress occurred varied at different strain rates, this observation is believed to be due to the surface roughness from processing rather than a material property.

Processing at an energy density of $0.056 \mathrm{~J} / \mathrm{mm}^{2}$ significantly reduced the flow stress; this energy density was achieved by reducing the scanning speed from $2500 \mathrm{~mm} / \mathrm{s}$ to $1500 \mathrm{~mm} / \mathrm{s}$. Energy densities, $0.03,0.034$ and $0.037 \mathrm{~J} / \mathrm{mm}^{2}$ were achieved by altering the laser power from 19 to $21 \mathrm{~W}$ and had a limited effect on the mechanical response.

Since there was no relationship between energy density and physical density, it seems that porosity is not the driving factor in determining the mechanical response within the aforementioned range of ED.

Other factors such as DPM that defines the completeness of melting within the microstructure may have a more significant influence on mechanical properties.

A deeper understanding of the relationship between energy density and rate dependency could provide an alternative insight into the role of the microstructure, whilst also allowing optimisation of process parameters for a particular strain rate environment.

\section{References}

[1] N. Hopkinson, R. Hague, P. Dickins, Rapid Manufacturing, Wiley, (2005)

[2] Kamperman, J.J. de Vlieger, Materials Science and Engineering, 435, 172 (2006)

[3] H. Zarringhalam, C. Majewski, N. Hopkinson, Rapid Prototyping J, 15, 126 (2009)

[4] R.D. Goodridge, C.J. Tuck, R.J.M. Hague, Progress in Materials Science, 57, 229, (2012)

[5] J.C. Nelson, phD Dissertation, Uni of Texas at Austin, Texas, (1993)

[6] C. Yan, Y. Shi, L. Hao, Polymer processing J, 4, 416 (2011)

[7] G.T. Gray III, ASM Handbook, Mechanical Testing and Evaluation' 8, 560, (2000)

[8] M.I. Okereke, C.P. Buckley, C.R. Siviour, Mechanics of Time-Dependent Materials, 16, 361, (2012)

[9] M. Boyce, R. Haward, The physics of glassy polymers, 508 (1997)

[10] Y. Wang, E.M. Arruda, Eng. Mater. Technol. J, 128, 551(2006)

[11] C.R. Siviour, S.M. Walley, W.G. Proud, N.F. Kamperman, J.J. de Vlieger, Materials Science and Engineering, 435, 172 (2006)

[12] E.W. Andrews, L.J. Gibson, M.F. Ashby, Acta Mater, 47, 2853 (1999)

[13] B. Caufield, P.E. McHugh, S. Lohfeld, Mater. Processing Technol. J, 182, 477 (2006) 